\title{
Vertical shift register using dipolar interaction in magnetic multilayers
}

\author{
Shin-Liang Chin ${ }^{1}$, Amalio Fernández-Pacheco ${ }^{1}$, Dorothée C. M. $C^{1}$. Petit \& Russell P. \\ Cowburn ${ }^{1}$. \\ ${ }^{1}$ Cavendish Laboratory, University of Cambridge, JJ Thomson Ave., Cambridge CB3 OHE, \\ United Kingdom.
}

A vertical shift register consisting of multi-layered ferromagnetic bars with in-plane magnetization is investigated numerically using macrospin simulations. These layers are anti-ferromagnetically coupled via dipolar interactions and their in-plane aspect ratio determines their anisotropy. A single data bit is represented by a magnetic kink soliton, which forms at the boundary of two anti-parallel domains with opposite phases. It can be propagated bi-directionally using an externally applied rotating magnetic field. The soliton propagation is dependent on the applied field strength, the magnetic anisotropy of the ferromagnetic layers and the dipolar coupling energies. For the device investigated here, the largest field range for soliton propagation is found to be from 35 Oe to $235 \mathrm{Oe}$ at a lateral aspect ratio of 1.33. The soliton is also subjected to edge effects where it can be either pinned or reflected rather than being expelled from the stack. It is found that by reducing the thickness of the edge layer, these effects can be reduced substantially. By reducing the thickness of the edge layer by $20 \%$, the field range in which the soliton is expelled increases by more than a factor of two.

\section{INTRODUCTION}

Increasing the areal density of data storage devices is becoming progressively difficult due to the trilemma of writability, thermal stability and signal-to-noise ratio ${ }^{1-3}$. The data storage industry is looking at shingled magnetic recording ${ }^{3}$, bit-patterned media ${ }^{4,5}$ and heat-assisted magnetic recording $^{6,7}$ as possible solutions. However, all these solutions still store data in a two-dimensional mesh. Moving into the third dimension could provide a real revolutionary increase in the areal density of magnetic storage devices ${ }^{8-12}$. This can be achieved by designing a vertical shift register that allows data to be transported out-of-plane from one cell to another. One such device is the magnetic ratchet we recently demonstrated ${ }^{9,13}$. This device transfers data in the form of a magnetic kink soliton through a magnetic multilayer with nearest-neighbor antiferromagnetic (AF) RudermanKittel-Kasuya-Yosida (RKKY) interactions and out-of-plane anisotropy. A soliton is formed at the boundary between two neighboring anti-parallel (AP) domains with opposite phases. It can be moved in a single direction synchronously with a global oscillating magnetic field. The possibility of realizing an all-spin-based device such as this is extensively investigated as an alternative to modern electronics to store energy and to process information ${ }^{14-16}$.

A version of such a vertical shift register which uses magnetic material with in-plane anisotropy, rather than out-of-plane, has also been designed using nearest neighbor RKKY interactions and intrinsic magnetocrystalline anisotropy ${ }^{11,12}$. This version of the device allows bidirectional propagation of solitons. The existence of solitons in such a thin film multilayer was demonstrated experimentally ${ }^{12}$, their stability was found to depend on the application of a magnetic field. As the lateral dimensions are reduced, dipolar interactions become substantial, which may interfere with the operation of the device ${ }^{17}$. In the case of layers magnetized out-of-plane, dipolar 
interactions between layers are ferromagnetic, and would have to be compensated for in order to maintain correct device operation upon downscaling. In the in-plane case, however, dipolar interactions between layers are AF and therefore have the potential to replace RKKY interactions and become the sole source of interlayer AF coupling. In the case of the system studied in Ref. 12 however, an increase of the effective AF coupling without the introduction of another source of anisotropy, such as shape anisotropy, could destabilize solitons.

In this paper, we propose a design for a multilayered soliton shift register which only uses dipolar interaction and in-plane shape anisotropy. Such a device can be realized due to the recent progress in nanowires fabrication techniques ${ }^{18-23}$. This device also has the added advantage that it can be fabricated using more commercially competitive methods such as electro-deposition ${ }^{21}$ and atomic layer deposition ${ }^{19,20,22}$, which are unlikely to produce interfaces of sufficient quality to support strong RKKY interactions. Sergelius et al. have shown that by using laser interference lithography to create nanochannel arrays, rectangular bars with high aspect ratios and with lateral dimensions similar to the ones described in this manuscript can be fabricated experimentally ${ }^{22}$.

\section{METHODOLOGY}

The structure investigated here consists of 20 layers of rectangular ferromagnetic bars equally spaced (Fig. 1(a)). The saturation magnetization $\left(M_{s}\right)$ was taken at a typical value for widely used soft in-plane materials $\left(1000 \mathrm{emu} / \mathrm{cm}^{3}\right)$. The bars are $5 \mathrm{~nm}$ thick $\left(t_{\mathrm{M}}\right), 60$ to $95 \mathrm{~nm}$ long and 60 $\mathrm{nm}$ wide. This creates a lateral aspect ratio of 1.00 to 1.58 , inducing a shape anisotropy with varying strength. The spacer thickness $\left(t_{s}\right)$ is $5 \mathrm{~nm}$. The propagation of the soliton is modeled using a macrospin description, which assumes that each layer is a single magnetic moment. The magnetization is constrained to rotate only in the plane of each layer since it is assumed that in practice the shape anisotropy will be sufficiently strong to keep the magnetization in-plane. MonteCarlo simulations are performed.

The dimensions are chosen to ensure near uniformity of the magnetization in individual layers, which is a necessary condition to the validity of the macrospin model. We have used micromagnetics simulations ${ }^{24}$ to check that this is indeed the case: when the magnetization is initialized in the single domain AP state and left to relax in zero field, the AP state remains with the magnetization of each layer fairly uniform. Furthermore, when each layer is initialized in a vortex state, the system also remains in a vortex state but with a higher energy than the AP state (see supplemental material ${ }^{25}$ ), demonstrating that the vortex state is only metastable. We have also performed full micromagnetic simulations of soliton propagation in a 20-layer stack with two different sets of dimensions and applied field strength. Although a significantly higher field was required than predicted by the macrospin model, correct soliton propagation was demonstrated. We believe that the quantitative discrepancy is due to the presence of configurational anisotropy ${ }^{26}$ in our nearly-uniform rectangular system and that the results described here are still qualitatively valid. This study is included in the supplemental material ${ }^{25}$.

\section{RESULTS AND DISCUSSIONS}

\section{A. The soliton}


A soliton is formed at the junction between two AP domains with opposite phase. Hence, at the center of a soliton are two magnetic layers with magnetization pointing in the same direction, which will henceforth be referred to as the soliton bilayer or the soliton layers. The soliton bilayer has a net magnetic moment. Unlike the layers in the rest of the bulk, which are stabilized into an AP state by neighbors on both sides, the soliton bilayer experience a stabilizing field from one side only, and destabilizing from the other. Therefore, these layers are the easiest to reverse. When one of the layers reverses, another soliton bilayer is formed at the neighboring layer. Thus, the soliton has propagated one layer. Because of the competition between the opposing stray fields from above and below, a soliton layer can, if the anisotropy is weak enough, splay at an angle. This breaks the symmetry around the soliton and gives it a chirality that is essential in ensuring a well-controlled propagation of the soliton under a globally applied rotating field ${ }^{11}$. The order in which the soliton bilayer switches, and therefore the propagation direction of the soliton, is determined by the sense of rotation of the field and the chirality of the soliton. The stability of the soliton also is influenced by the interplay between shape anisotropy and dipolar interactions. If the anisotropy energy is much larger than the coupling energy, the solitons will point strictly along the easy axis and lose chirality. On the other hand, if the coupling energy is much larger than the anisotropy, the solitons will be expelled from the stack because the AP configuration is more stable. The spacer thickness influences the dipolar interactions between the layers, whilst the aspect ratio influences both the dipolar interactions and the shape anisotropy. If the spacer distance and the aspect ratio are carefully chosen, a stable soliton can be initialized in the middle of the stack. In order to visualize the soliton and its propagation along the stack, we define a quantity for each layer, the AP phase parameter, $\Phi_{n}=(-1)^{n} \cdot \cos \theta_{n}{ }^{11,12}$, where $\theta_{n}$ is the angle between the magnetization of the $n$-th layer and the easy axis. $\Phi_{n}$ changes sign at the center of a soliton, as shown in Fig. 1(b). Thus, the sum of $\Phi_{n}$ across the entire stack gives an indication of the position of the soliton in the stack. If the sum of $\Phi_{n}$ is zero, the soliton is located exactly in the middle of the stack. For every layer the soliton moves, the sum of $\Phi_{n}$ changes by two. The injection of solitons in RKKY-coupled devices has already been experimentally demonstrated in both in-plane ${ }^{12}$ and out-of-plane materials ${ }^{13}$. Here, the injection of the soliton into this in-plane dipolar-coupled device is not explicitly investigated but instead a soliton is initialized in the middle of the stack. A global rotating field, either clock-wise (CW) or counter clock-wise (CCW) when seen from the top, is then applied to propagate the soliton.

\section{B. Propagation in the bulk}

The principles of soliton propagation are outlined here. Fig. 2(a) to (d) shows four snapshots of the soliton propagation in bulk during different phases of the rotating field. The aspect ratio used here Is 1.17 and the magnitude of the rotating field is 120 Oe. Due to the splaying of the angle between the layers that form the soliton, a CW rotating field will first be aligned with the bottom of the soliton layers, labelled A in Fig. 2(a). However, in order for layer A to rotate CW with the field, it needs to go via a state where it is fully parallel to layer B. This is energetically very costly and does not happen if the field is too low (Fig. 2(b)). As the externally applied field continues its CW rotation, it now aligns with $B$, which is more easily reversed than $A$ (Fig. 2(C)). As the magnetization of layer $B$ reverses, a soliton with the same chirality is now between $B$ and the layer above it, labelled $C$ (Fig. 2(d)). Thus, the soliton has moved one layer up. At every half a cycle, the rotating field aligns itself with a different layer, creating the upward motion of the soliton in the stack. The existence of the chirality or the splaying of the angle is essential. Without it, the reversal of the top or bottom layer of bilayer will be indistinguishable in energy thus resulting in an uncontrolled propagation direction 
of the soliton. Fig. 2(e) shows the movement of the soliton, in terms of sum of $\Phi_{n}$, as it moves upwards using a CW field.

A phase diagram showing the different modes of propagation as a function of aspect ratio and applied field strength is presented in Fig. 3. Here, the shape anisotropy is modified through the aspect ratio of the ferromagnetic layers. For aspect ratios of less than 1.07, a soliton cannot be stably contained within the 20-layer stack at zero field. For aspect ratios of 1.07 and above, various propagation behaviors of the soliton exist. When the applied field is too small, the soliton is unable to propagate (yellow). On the other hand, if the applied field is too large, the edge layers nucleate (red). For large aspect ratios where the shape anisotropy is the strongest, a large enough applied field will cause the two soliton layers to point exactly along the easy axis when the field is also along the easy axis. The soliton chirality is momentarily lost and which layer switches first is not determined anymore, causing the soliton to move randomly (dark blue). In between these extremes, the soliton propagates synchronously with the applied field (light blue). The details within this region will be described in the next section.

\section{Propagation at the edge}

The edge layers are subjected to long range dipolar interactions from one side only. Therefore, a soliton at the edge will behave differently than in the bulk. As the soliton approaches the edge, it may not be successfully expelled out of the stack but instead may be reflected or pinned, depending on the aspect ratio of the structure and the strength of the applied field (see the light blue region in Fig. 3). There is only a small aspect ratio range where the soliton can propagate without pinning or reflecting, which is between 1.07 and 1.23.

Fig. 4(a) to (d) shows snapshots of the pinning process, which occurs at low applied fields. The aspect ratio used here is 1.17 and the magnitude of the applied rotating field is 60 Oe. Consider a soliton at layers $A B$ approaching an edge layer, labelled C in Fig. 4(a). Layer C, being closer to the edge, does not experience as strong a stabilizing field from layers above. Its interaction with layer $B$ therefore dominates and layer $C$ remains more antiparallel to layer $B$ than if $C$ was a bulk layer. The flux closure configuration between $B$ and $C$ reduces the ability of $B$ to couple with the rotating field. The rotating field needs to reverse $B$ in order to move the soliton into the edge layer and then expel it, but if the field strength is too low, B is left lagging behind the applied field and does not reverse (Fig. 4(b)). As the field completes its half cycle rotation, the magnetization of layer $A$ is pulled towards it and begins reversing instead (Fig. 4(c)). The chirality of the field is the wrong one for $A$ to complete its reversal and $A$ switches back. The system has gone back to its original configuration (Fig. $4(d)$ ), i.e. the soliton is pinned. Fig. 4(e) shows the movement of the soliton during this process. The multiple dips are due to the sudden simultaneous releasing of $B$ and pulling of $A$ by the rotating field happening over and over.

At higher field strengths, a reflection of the soliton may be observed instead. When the direction of the applied field aligns with the direction of the soliton bilayer and the easy anisotropy axis, the splaying angle of the soliton layers reduces. For high enough applied fields, the angle may reduce to zero, effectively causing the chirality of the soliton to disappear and symmetry to be reestablished. In the bulk of a stack, this causes the random propagation of solitons mentioned earlier (dark blue area in Fig. 3). The case of edge layers is illustrated in Fig. 5. Consider a soliton at layers $A B$ approaching edge layer $C$ from below (Fig. 5(a)). In this case, layer B is also tightly coupled 
to $\mathrm{C}$, the layer immediately above it. Just like in the case of soliton pinning, this makes it more difficult for B to couple with the applied field. Because the chirality is lost or nearly lost, it is actually easier for layer A to rotate than for layer B (Fig. 5(b)), causing the soliton to move one layer down, effectively flipping its chirality (Fig. 5(C)). When moving away from the edge, the new soliton chirality is preserved under the same applied field strength. Subsequent rotation of the field will cause the soliton to move in the opposite direction, i.e. the soliton is reflected by the edge of the stack (Fig. 5(d)). Fig. 5(e) shows the motion of the soliton when this happens. The figure shows the soliton bouncing back and forth between layers 2 and 18 .

The phase diagram depends on the interplay between the AF dipolar coupling and the inplane anisotropy. When a different saturation magnetization $\mathrm{M}_{\mathrm{S}}$ or different dimensions (width $\mathrm{w}$, magnetic thickness $t_{M}$ or spacer thickness $t_{S}$ ) are used, the borders of the phase diagram may shift but we believe that its overall shape will remain the same. To verify this, we calculated the slice of the phase diagram at an aspect ratio of 1.2 as a function of $M_{s}, t_{M}$ and $t_{s}$, for a $\pm 20 \%$ variation of these parameters around the values used in Fig. 3. The results are shown Fig. 6. As $M_{s}$ increases between 800 and $1200 \mathrm{emu} / \mathrm{cc}$, the soliton propagation region increases by $52 \%$, essentially because of an increase in nucleation field with increasing $M_{S}$ (Fig. 6(a)). Fig. 6(b) shows that as the magnetic layer thickness increases between 4 and $6 \mathrm{~nm}$, not only does the soliton propagation region increase, but also the soliton is more likely to be expelled than pinned at the edge. In this case also, the boundary between propagation and no propagation remains unchanged. Fig. 6(c) shows the effect of increasing the spacer thickness. In that case, the region where the soliton is able to propagate remains fairly unchanged but the soliton becomes increasingly likely to be pinned at the edge. Fig. 6(d) shows how the change of the lateral size affects the propagation modes. Maintaining the same $A R$ of 1.2 , the width of the magnetic layer is varied by $\pm 20 \%$. When the width is reduced, the nucleation field is increased. At very small size, i.e. when the width and length are $48.0 \mathrm{~nm}$ and 57.6 $\mathrm{nm}$ respectively, the nucleation field is significantly increased to beyond 250 Oe. At this size, the random and reflection phases start to appear.

By using an optimization algorithm, optimum parameters would be found that would result in a larger field range for soliton propagation to occur without pinning or reflection, but this is beyond the scope of this paper.

\section{Modifying edge effects}

Since pinning and reflection are due to edge effects, they can be prevented by modifying the properties of the edge layers. Both the pinning and reflection of the soliton at the edge are due to the strong coupling of the soliton with the edge layer. Reducing this coupling can be done by reducing the thickness of the edge magnetic layer. Fig. 7(a) shows the field ranges for the different propagation modes for two aspect ratios when reducing the thickness of the edge layer by $1 \mathrm{~nm}$ (or $20 \%)$.

In the case for the 1.20 aspect ratio, a $1 \mathrm{~nm}$ reduction in the edge layer thickness increases the range of field for which the soliton is neither pinned nor reflected from $125-185$ Oe to $60-195$ Oe, which is more than a two-fold increase. In the case of the 1.33 aspect ratio, the same $1 \mathrm{~nm}$ reduction in the thickness of the edge layer opens up a gap of fields (55-165 Oe) for which solitons can be expelled through the edge without getting pinned or reflected. 


\section{CONCLUSIONS}

A vertical shift register using only dipolar interaction is described here. Unlike in the RKKYcoupled magnetic ratchet device where dipolar interactions may have undesirable effects when the device is scaled down, the device described here actually uses the long range dipolar interactions as a constitutive ingredient. A magnetic kink soliton can be synchronously propagated using a global rotating field. We have presented a simulation of the phase diagram of a $60 \mathrm{~nm}$ wide device, with varying lengths to cover aspect rations between 1 and 1.58, showing different propagation behaviors depending on the aspect ratio of the structure and the strength of the applied magnetic field.

In the region of the phase diagram where bulk propagation occurs, different behaviors may arise at the edge of the stack. As the soliton approaches the edge, it may be either expelled, pinned or reflected. Whilst pinning occurs at low field strength, reflection occurs at high fields where the chirality of the soliton is significantly weakened by the application of the field. The field range where soliton propagation occurs without pinning or reflection at the edges takes place only in a narrow range of aspect ratio of $1.07-1.23$. The widest range of applied fields where expulsion occurs is 65 175 Oe at an aspect ratio 1.17. This can be improved by reducing the thickness of the edge layer. We show an example where reducing the thickness of the edge layers by $20 \%$ increases the field range for correct operation by a factor of 2 .

The phase diagram shows that this device is quite tolerant to geometrical variations, which allows it to be fabricated using more commercially competitive methods such as electrodeposition and atomic layer deposition.

\section{ACKNOWLEDGEMENTS}

This project has received funding from the European Union Seventh Framework Programme (FP7/2007-2013) under grant agreement no. 309589 (M3d), as well as the Seventh Framework Programme ERC Contract No. 247368 (3SPIN). AFP acknowledges funding from an EPSRC Early Career Fellowship, EP/M008517/1 and from the Winton Foundation. Additional data related to this publication is available at the www.repository.cam.ac.uk/handle/1810/252622 data repository. 


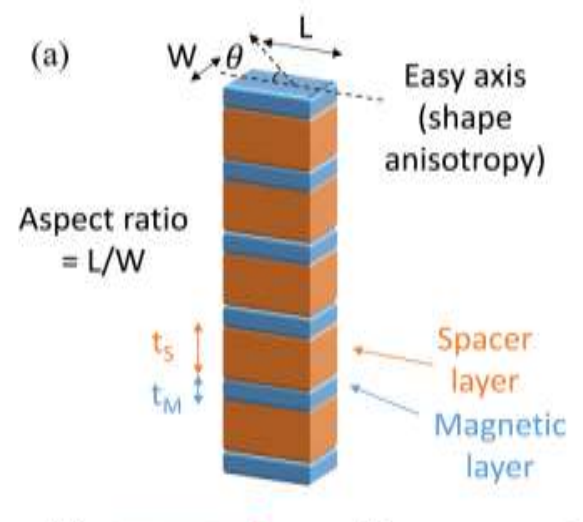

(b)

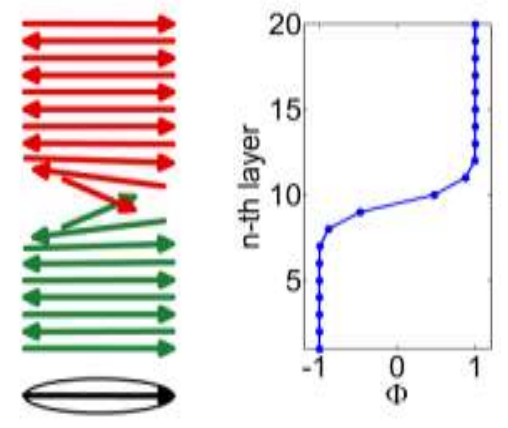

Fig. 1. (a) Schematic of the vertical shift register. (b) In-plane magnetization direction of the rectangular bar stack and the phase parameter $\Phi_{n}$. The black arrow above the stack shows the direction of the rotating field. The asterisk shows the position of the soliton. 


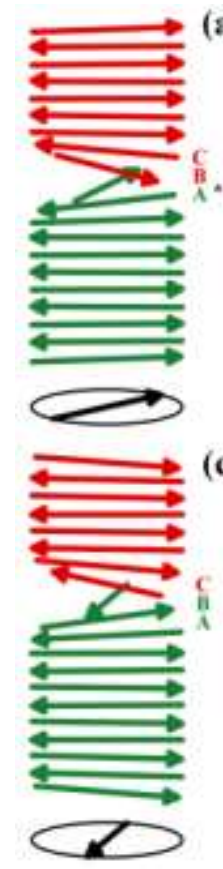

(a)
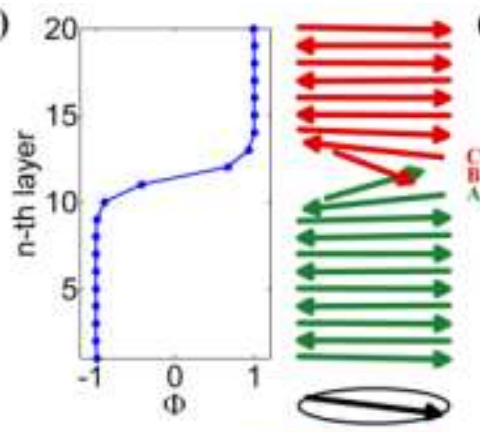

(b)

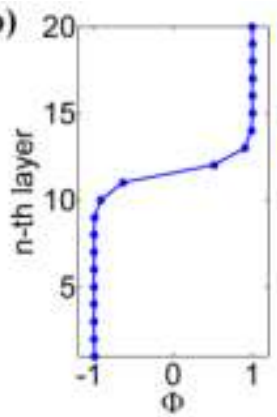

(c)
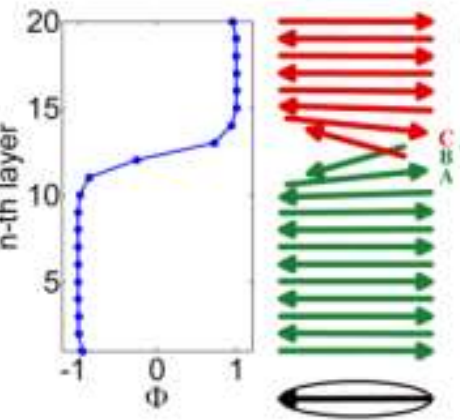

(e) 24

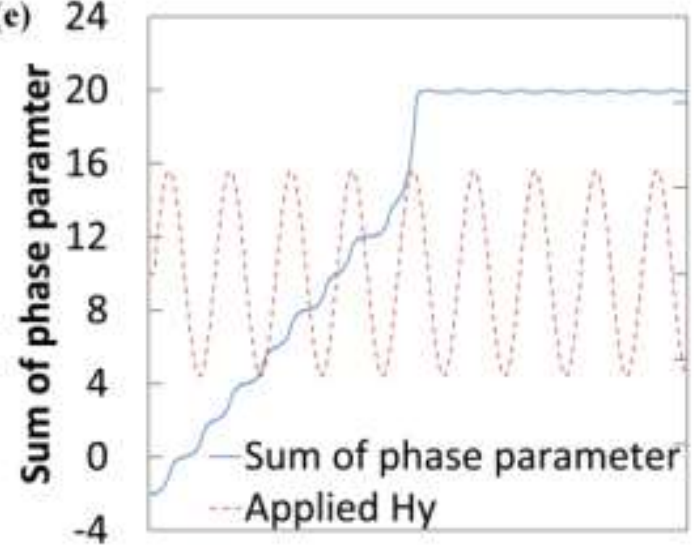

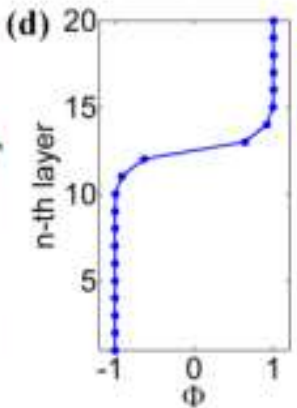

300

\section{0}

100

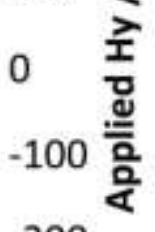

$-200$

$-300$

Fig. 2. Bulk soliton propagation. Initially, the center of the soliton is located at bilayer AB. (a) As the field rotates in the $\mathrm{CW}$ direction (as seen from the top), it first aligns itself with layer $A$.

However, it is more energetically favorable to reverse layer B, as shown in (b) to (c). (d) At the end of the half cycle, the center of the soliton is now at bilayer BC. (e) Time evolution of the sum of the phase parameter for a soliton propagating and eventually expelled at the edge. The right $y-$ axis shows the $y$ component of the rotating field. 


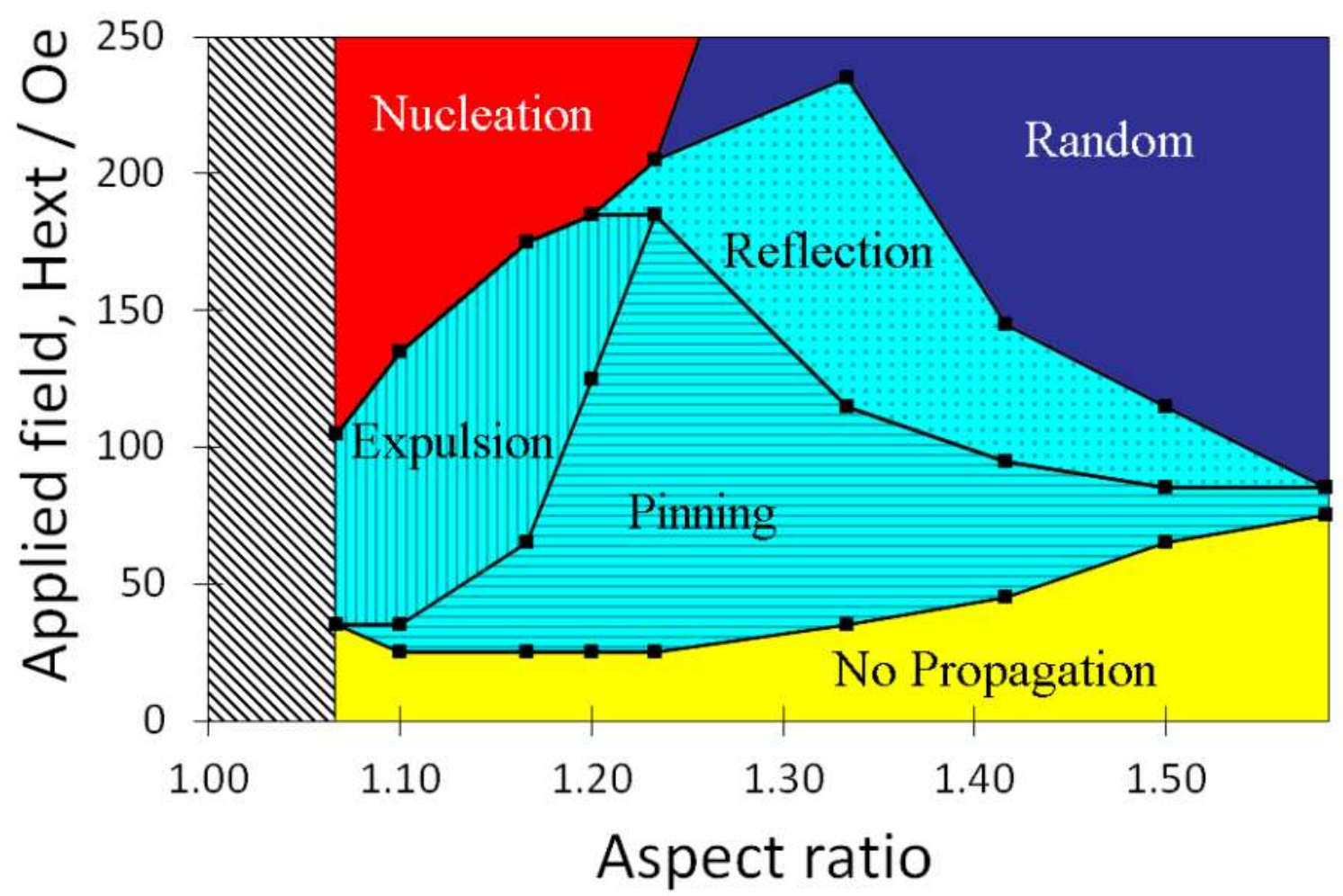

Fig. 3. Phase diagram for the different soliton propagation behaviors. When the applied field is too high, the edges reverse, this is the nucleation regime (red). When the field is too low, the soliton does not propagate (yellow). At high aspect ratios and high applied fields, the soliton propagation direction changes randomly (dark blue). At intermediate fields and aspect ratios, the soliton propagates correctly in the bulk of the stack (light blue). However, due to the edge effects, there can be pinning, reflection or expulsion. At aspect ratios less than $\mathbf{1 . 0 7}$ the soliton is not stable under zero field. 

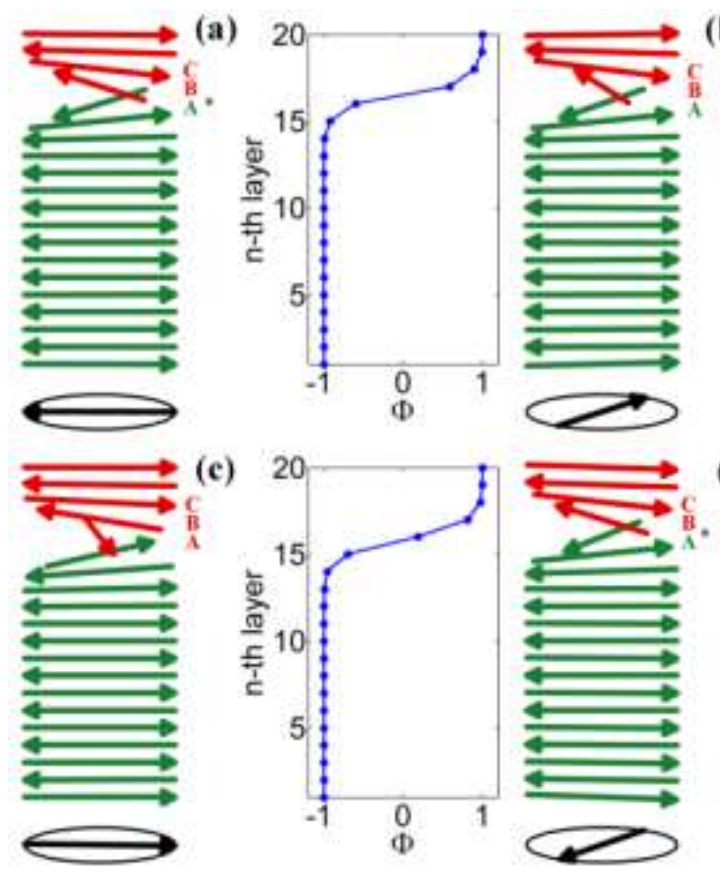

(b)

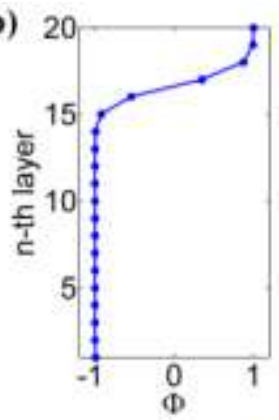

(c)


(e) 16

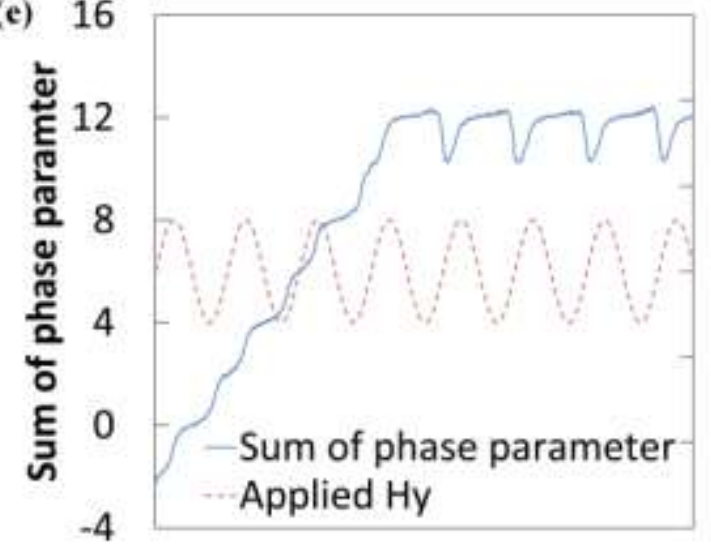



300

200



Fig. 4. Mechanism of soliton pinning at the edge. (a) A soliton approaches layer $C$ from below. (b) Layer B is unable to align itself with the field and thus is now lagging behind. (c) and (d) show that as the field rotates back to the left, it aligns with the bottom layer of the soliton again, and the whole process repeats. (e) Time evolution of the sum of the phase parameter for a soliton pinned at the edge. The dips show repeated attempts by the soliton to go past the edge, but it remains pinned at the second layer from the top. The right $y$-axis shows the $y$ component of the rotating field. 

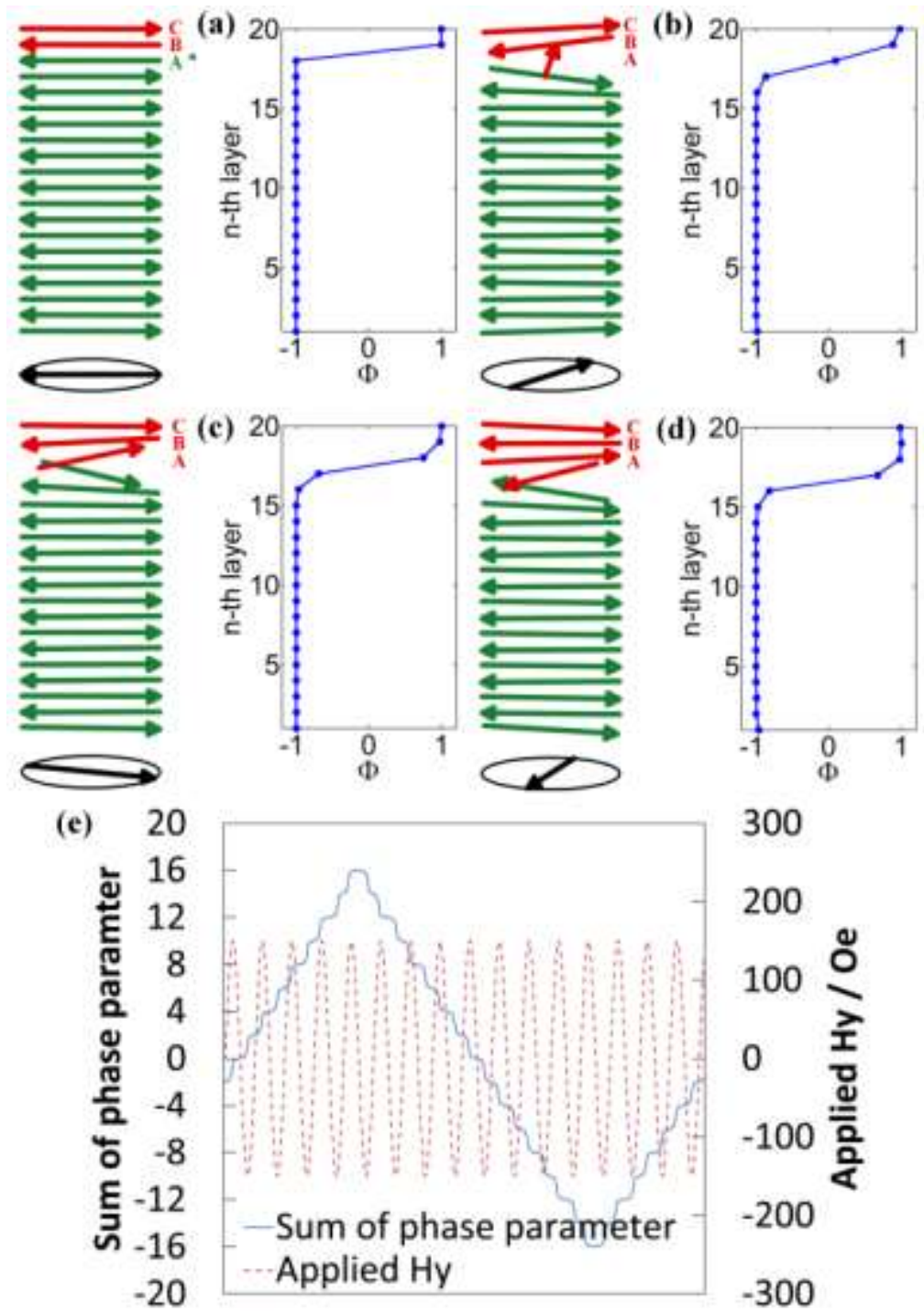

Fig. 5. Mechanism of soliton reflection at the edge. (a) As the field aligns with the easy anisotropy axis and the direction of the soliton layers, the chirality is momentarily reduced. (b) Layer $A$ follows the field, rather than layer B. (c) and (d) As the next half cycle begins, the chirality of the soliton is reversed, the soliton propagates in the opposite direction. (e) Time evolution of the sum of the phase parameter for a soliton reflected off the edge. The right $y$-axis shows the $y$ component of the rotating field. 

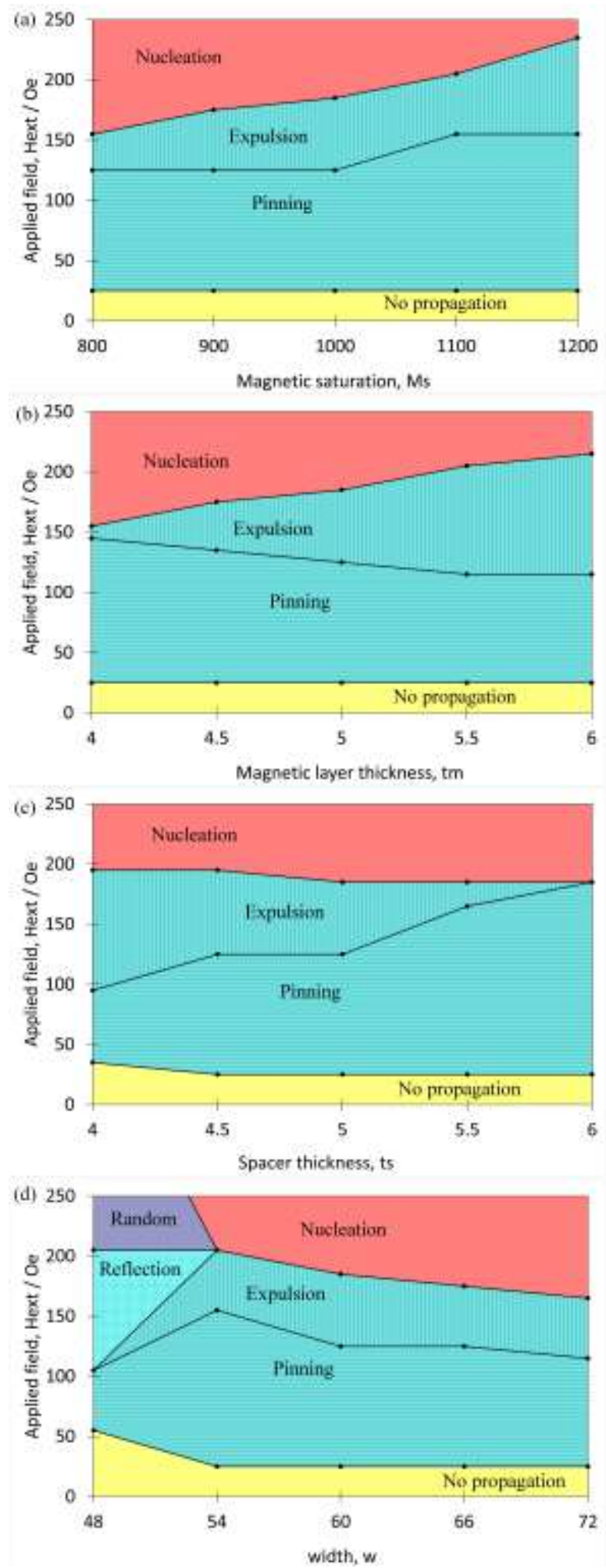

Fig. 6. The change of propagation modes as function of the parameters Ms, tm, ts and lateral size (changing width and length with a fixed AR). 
(a)

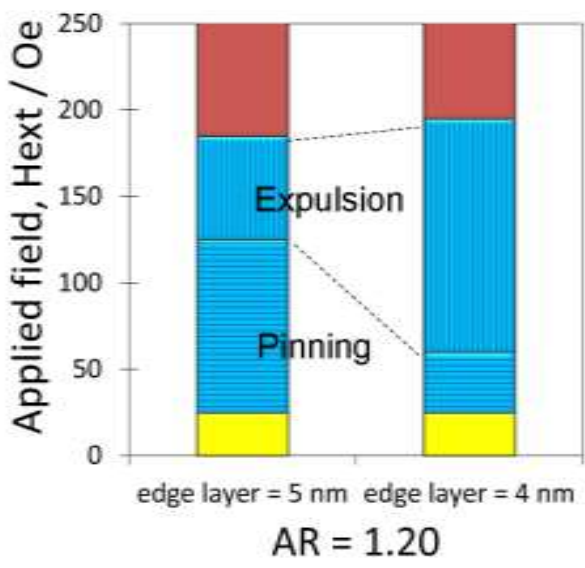

(b)

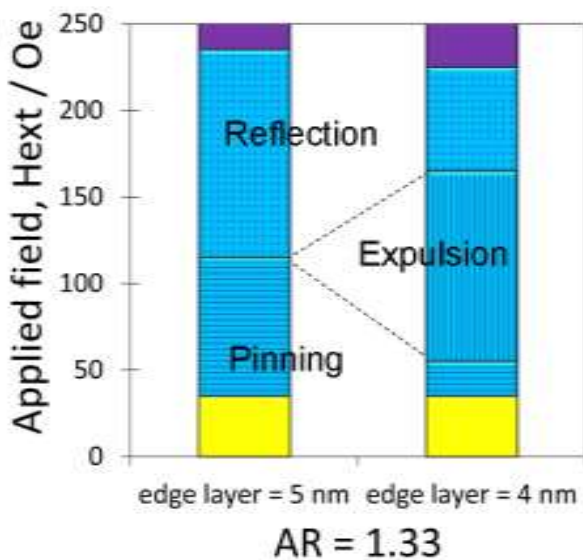

$\square$ Nucleation

Expulsion

目Pinning

$\square$ No

propagation

Random

Reflection

Expulsion

믈 Pinning

$\square$ No

propagation

Fig. 7. (a) and (b) Effect of reducing the thickness of the edge magnetic layers from $5 \mathrm{~nm}$ to $4 \mathrm{~nm}$, i.e. by $20 \%$, on two different aspect ratios 1.20 and 1.33 . The range where expulsion occurs (green) increases significantly when the thickness of the edge layer is modified. 


\section{$\underline{\text { References }}$}

${ }^{1}$ A. Moser, K. Takano, D.T. Margulies, M. Albrecht, Y. Sonobe, Y. Ikeda, S. Sun, and E.E. Fullerton, J. Phys. Appl. Phys. 35, R157 (2002).

2 D. Weller and A. Moser, IEEE Trans. Magn. 35, 4423 (1999).

${ }^{3}$ R. Wood, IEEE Trans. Magn. 36, 36 (2000).

${ }^{4}$ C. Ross, Annu. Rev. Mater. Res. 31, 203 (2001).

${ }^{5}$ S.Y. Chou, Proc. IEEE 85, 652 (1997).

${ }^{6}$ R.E. Rottmayer, S. Batra, D. Buechel, W.A. Challener, J. Hohlfeld, Y. Kubota, L. Li, B. Lu, C. Mihalcea, K. Mountfield, K. Pelhos, C. Peng, T. Rausch, M.A. Seigler, D. Weller, and X. Yang, IEEE Trans. Magn. 42, 2417 (2006).

${ }^{7}$ M.H. Kryder, E.C. Gage, T.W. McDaniel, W.A. Challener, R.E. Rottmayer, G. Ju, Y.-T. Hsia, and M.F. Erden, Proc. IEEE 96, 1810 (2008).

${ }^{8}$ R.P. Cowburn, in 2011 Int. Conf. Electromagn. Adv. Appl. ICEAA (IEEE, 2011), pp. 981-981.

${ }^{9}$ R. Lavrijsen, J.-H. Lee, A. Fernández-Pacheco, D.C.M.C. Petit, R. Mansell, and R.P. Cowburn, Nature 493, 647 (2013).

${ }^{10}$ S.S.P. Parkin, M. Hayashi, and L. Thomas, Science 320, 190 (2008).

${ }^{11}$ D. Petit, R. Mansell, A. Fernández-Pacheco, J. Lee, and R.P. Cowburn, in VLSI Circuits Emerg. Appl., edited by T. Wojcicki (CRC Press, 2014), pp. 275-294.

${ }^{12}$ A. Fernández-Pacheco, D. Petit, R. Mansell, R. Lavrijsen, J.H. Lee, and R.P. Cowburn, Phys. Rev. B 86, 104422 (2012).

${ }^{13}$ R. Lavrijsen, D.C.M.C. Petit, A. Fernández-Pacheco, J. Lee, M. Mansell, and R.P. Cowburn, Nanotechnology 25, 105201 (2014).

${ }^{14}$ B. Behin-Aein, D. Datta, S. Salahuddin, and S. Datta, Nat. Nanotechnol. 5, 266 (2010).

${ }^{15}$ M. Menzel, Y. Mokrousov, R. Wieser, J.E. Bickel, E. Vedmedenko, S. Blügel, S. Heinze, K. von Bergmann, A. Kubetzka, and R. Wiesendanger, Phys. Rev. Lett. 108, 197204 (2012).

${ }^{16}$ E.Y. Vedmedenko and D. Altwein, Phys. Rev. Lett. 112, 017206 (2014).

17 J.H. Lee, D. Petit, R. Lavrijsen, A. Fernández-Pacheco, R. Mansell, and R.P. Cowburn, Appl. Phys. Lett. 104, 232404 (2014).

${ }^{18}$ A. Fernández-Pacheco, L. Serrano-Ramón, J.M. Michalik, M.R. Ibarra, J.M. De Teresa, L. O’Brien, D. Petit, J. Lee, and R.P. Cowburn, Sci. Rep. 3, (2013).

${ }^{19}$ C. Bae, R. Zierold, J.M. Montero Moreno, H. Kim, H. Shin, J. Bachmann, and K. Nielsch, J. Mater. Chem. C 1, 621 (2013).

${ }^{20}$ K. Pitzschel, J. Bachmann, J.M. Montero-Moreno, J. Escrig, and D. Görlitz, Kornelius Nielsch, Nanotechnology 23, 495718 (2012).

${ }^{21}$ A. Ramazani, M. Ghaffari, M.A. Kashi, F. Kheiry, and F. Eghbal, J. Phys. Appl. Phys. 47, 355003 (2014).

${ }^{22}$ P. Sergelius, J.M.M. Moreno, W. Rahimi, M. Waleczek, R. Zierold, D. Görlitz, and K. Nielsch, Nanotechnology 25, 504002 (2014).

23 J.M. Montero Moreno, M. Waleczek, S. Martens, R. Zierold, D. Görlitz, V.V. Martínez, V.M. Prida, and K. Nielsch, Adv. Funct. Mater. 24, 1857 (2014).

${ }^{24}$ M.J. Donahue and D.G. Porter, OOMMF User's Guide, Version 1.0 (National Institute of Standards and Technology, Gaithersburg, MD, 1999).

${ }^{25}$ See supplemental material at [URL will be inserted by AIP] for validation of macrospin model using micromagnetics simulation.

${ }^{26}$ R.P. Cowburn and M.E. Welland, Phys. Rev. B 58, 9217 (1998). 
(a)

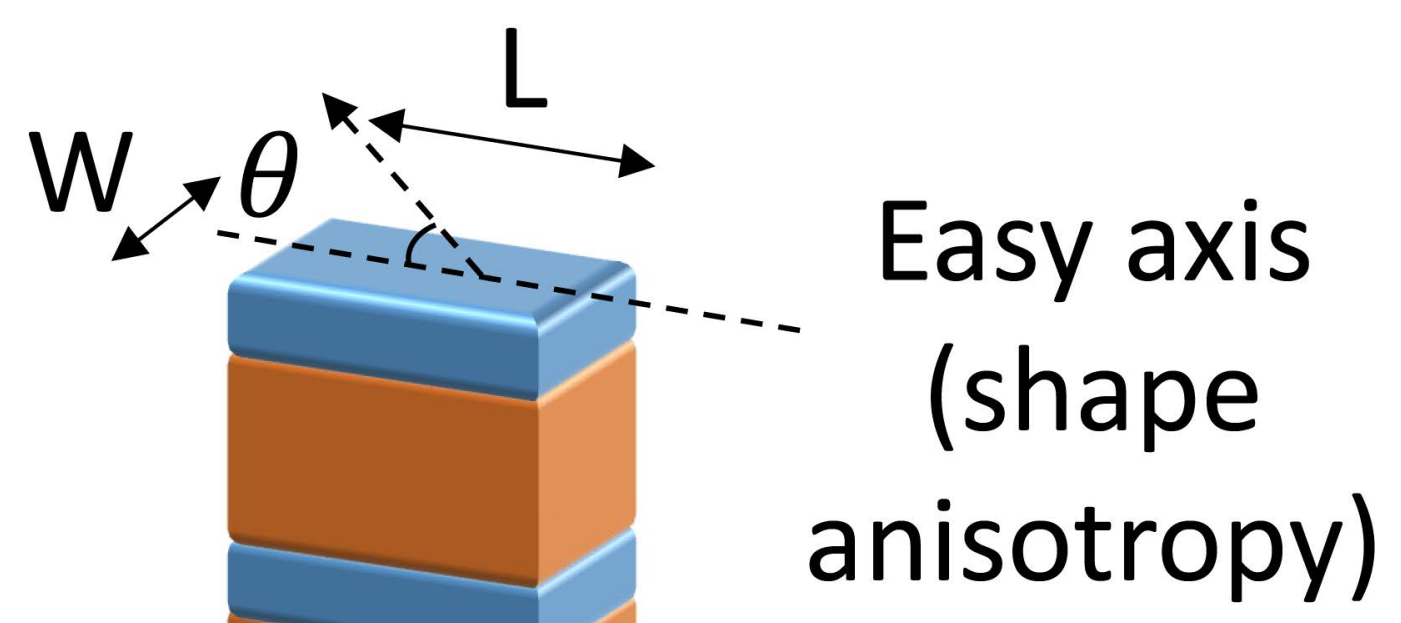

Aspect ratio

$$
=\mathrm{L} / \mathrm{W}
$$
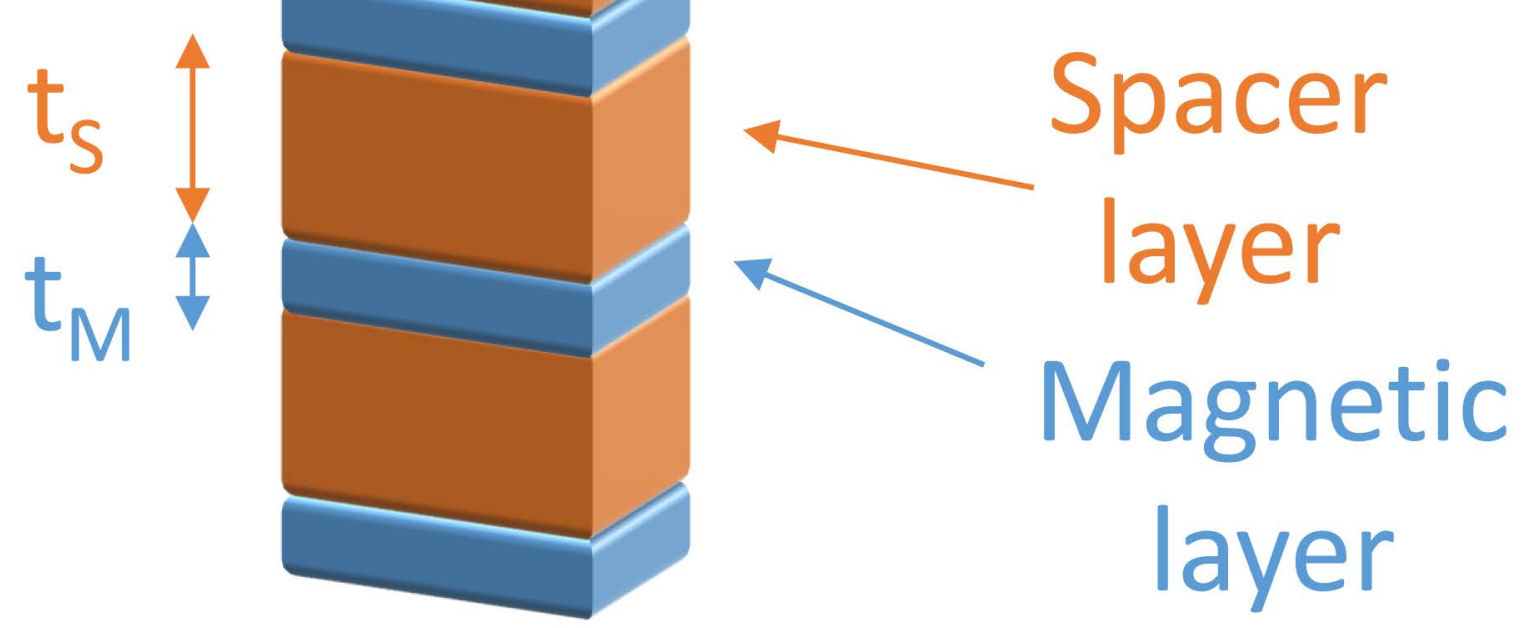

(b)
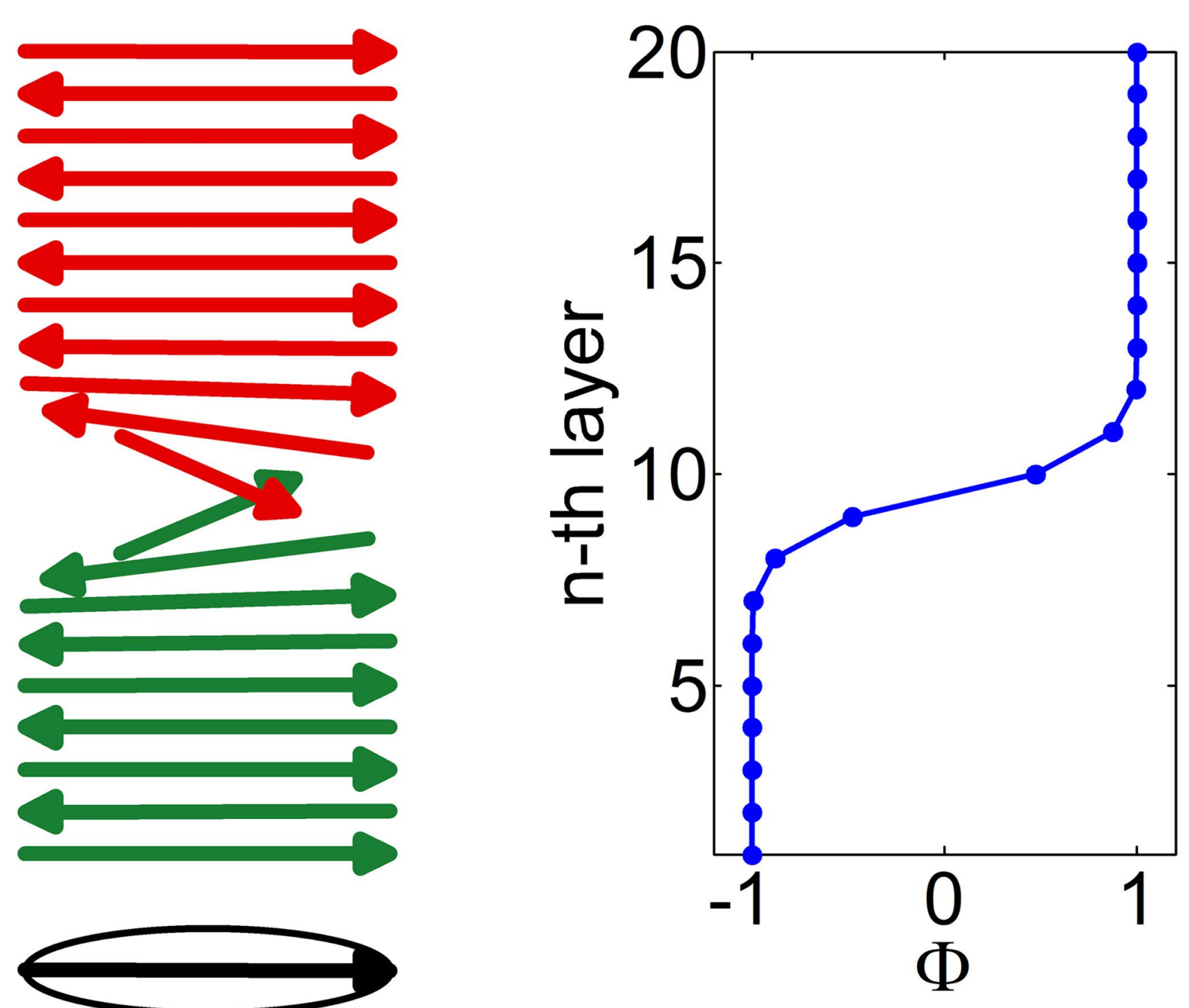



(d)

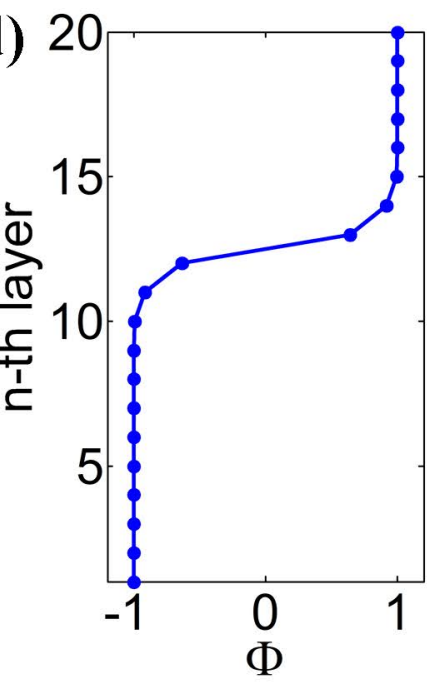

(e) 24

300






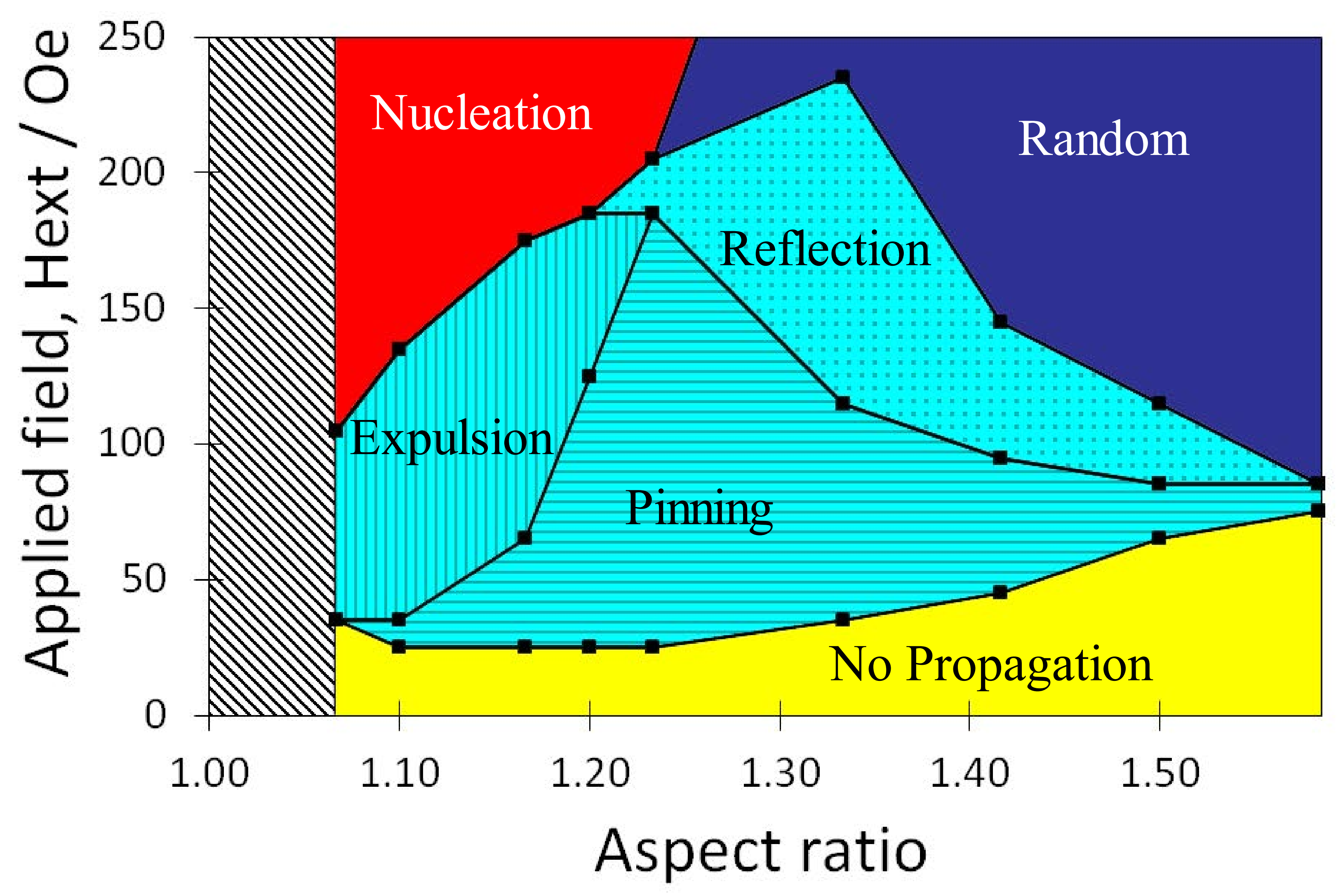



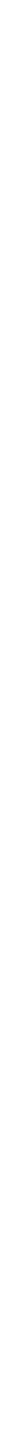

(c)
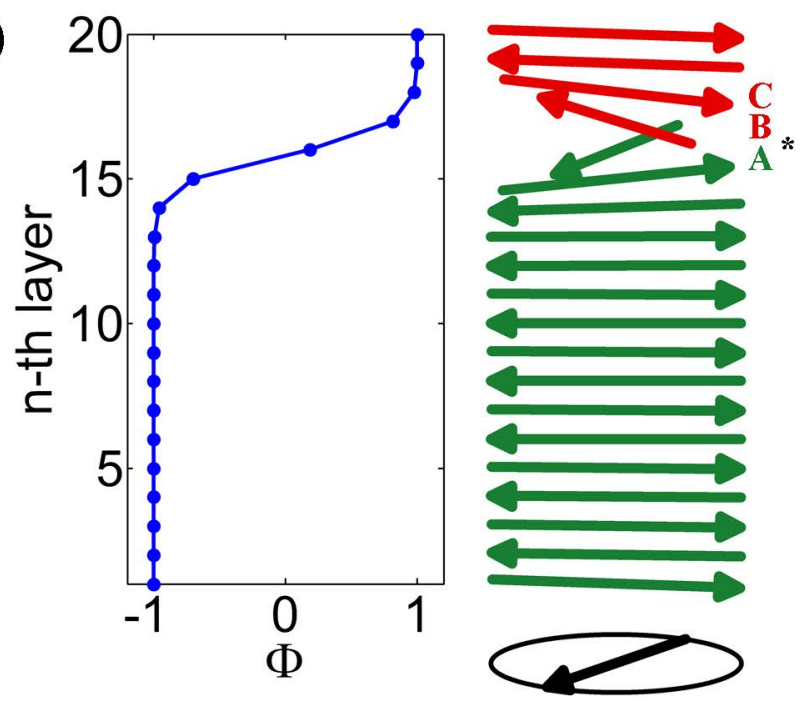

(d)

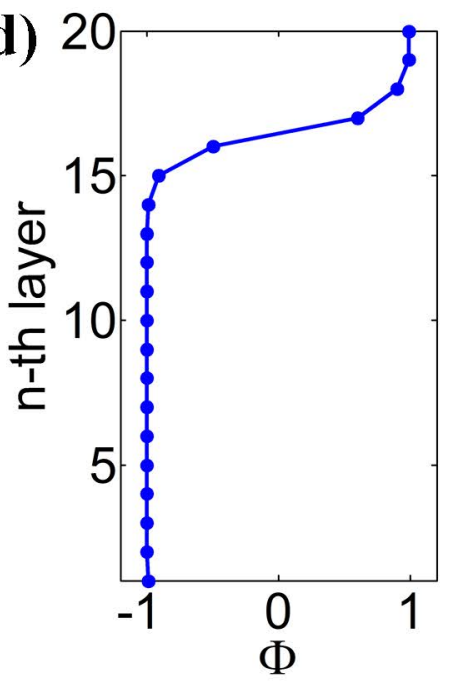

300

(e) 16

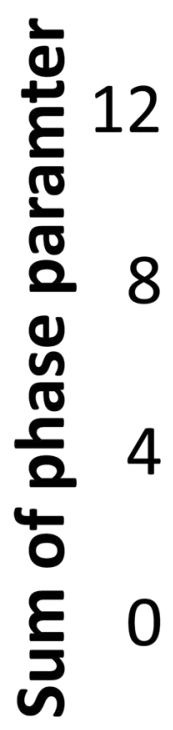

Sum of phase parameter-

$-200$ Applied Hy

200

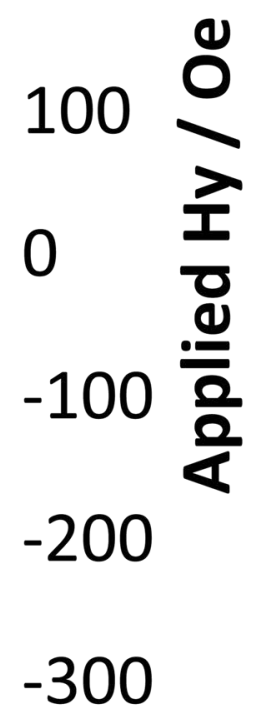



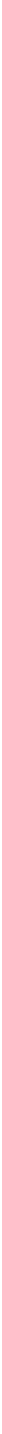

(d)

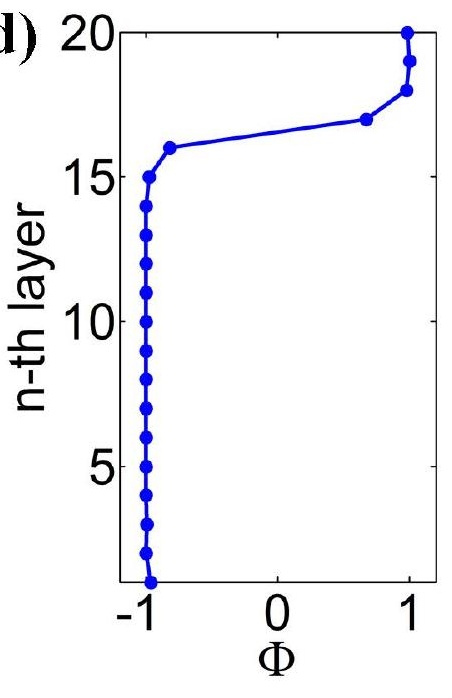

300

(e) 20

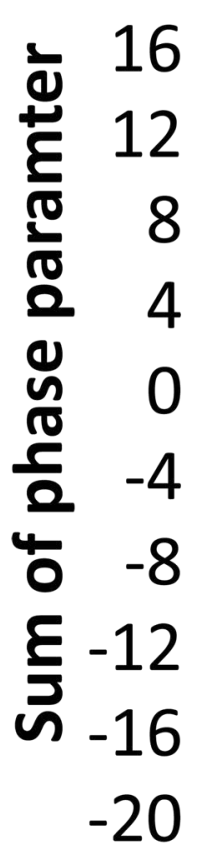

200



$-200$ 


\section{(a) oิ $^{250} \square$}

\section{四 Expulsion}

Expulsion

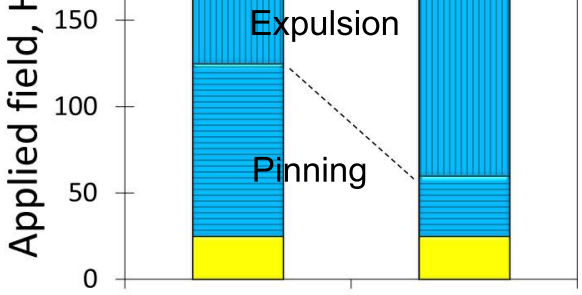

edge layer $=5 \mathrm{~nm}$ edge layer $=4 \mathrm{~nm}$

$$
A R=1.20
$$

(b) $\overbrace{}^{250}$

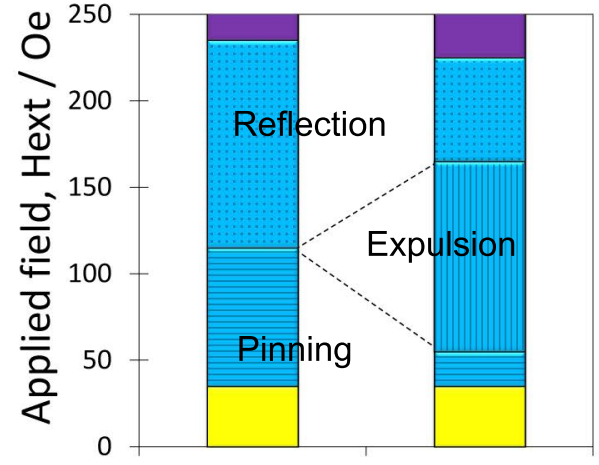

edge layer $=5 \mathrm{~nm}$ edge layer $=4 \mathrm{~nm}$

$$
A R=1.33
$$

$\square$ Random

Reflection

四 Expulsion

目Pinning

No

propagation 